\title{
Die "Wilden" und das Völkerrecht
}

\author{
Von Dieter Dörr
}

Der Titel1 mag bei manchen Zuhörem zunächst Verwunderung auslösen. Jedoch spielen die sogenannten Wilden im Zusammenhang mit der europäischen Expansion und der damit verbundenen Entwicklung des modemen Völkerrechts eine nicht unerhebliche Rolle. Die Figur des bzw. der Wilden diente dabei, wie noch zu zeigen sein wird, dazu, die Herrenlosigkeit der überseeischen Gebiete zu begründen und damit die Eroberungen der Europäer völkerrechtlich zu rechtfertigen.

\section{Die Wilden als Rechtsbegriff}

Die Wilden sind im Völkerrecht des 18. und 19. Jahrhunderts als Rechtsbegriff durchaus geläufig. So spricht schon Kant in seiner Schrift "Zum ewigen Frieden"2 im Zusammenhang mit dem Völkerrecht im zweiten Definitivartikel die Wilden ausdrücklich an. Diese befinden sich nach seiner Auffassung noch im Zustand der gesetzlosen Freiheit, balgen sich deshalb lieber unaufhörlich, als sich einem gesetzlichen, von ihnen selbst zu konstituierenden Zwange zu unterwerfen. Nach seiner Ansicht ziehen die Wilden, z.B. die amerikanischen Indianer, die tolle Freiheit der vernünftigen vor und müssen deshalb wegen ihrer Rohigkeit, Ungeschliffenheit mit tiefer Verachtung betrachtet werden. Auf der anderen Seite beklagt Kant, daß sich auch die vermeintlich gesitteten europäischen Völker gleichermaßen verworfen verhalten. Der Unterschied zwischen den europäischen Wilden und den amerikanischen bestehe hauptsächlich darin, daß manche amerikanischen Stämme von ihren Feinden gänzlich gegessen würden, während die europäischen Wilden ihre Uberwundenen besser zu benutzen wüßten. Diese recht merkwürdigen Ansichten über die Lebensweisen der amerikanischen Indianer und anderer außereuropäischer Völker sind nicht etwa für Kant typisch. Gerade Kant hat an anderer Stelle dezidiert gegen die Theorie der Herrenlosigkeit der überseeischen Gebiete und das schrankenlose Okkupationsrecht der

1 Diese Abhandlung gibt die Antrittsvorlesung wieder, die der Verfasser am 30.11.1989 an der Universität Hamburg gehalten hat. Der Verfasser hat zu dieser Thematik, allerdings auf die USA beschränkt, bereits die Abhandlung Die "Indian Nations and Tribes" in Nordamerika und das Völkerrecht, JÖR n.F. 36 (1987), 489 ff. verfaßt.

2 Vgl. Immanuel Kant, Schrift zum Ewigen Frieden, Gesammelte Werke, hrsg. von Wilhelm Weischedel, Bd. XI, S. 206 f. 
Europäer Stellung genommen. ${ }^{3}$ Jedoch haben solche grob fehlerhaften Auffassungen über das Leben der außereuropäischen Völker große Auswirkungen gehabt und die Theorie der herrenlosen Gebiete nachhaltig beeinflußt. Zunächst muß aber aufgezeigt werden, wieso die Rechtsfigur des Wilden im Völkerrecht Bedeutung gewann.

Vor den großen Entdeckungen des 15. Jahrhunderts spielt sich der Rechtsverkehr zwischen den verschiedenen Gemeinwesen innerhalb eines relativ fest umgrenzten Bereiches ab. Dabei bilden die christlichen Reiche in Europa den Kem und pflegen untereinander vielfältige rechtliche Beziehungen. Daneben bestehen die islamischen Reiche. Für den Rechtsverkehr zwischen diesen beiden Bereichen gelten im Mittelalter besondere Regelungen, die vielfältige Einschränkungen und Verbote enthalten. Dabei bildet der griechisch-byzantinische Kulturkreis die Klammer zwischen den beiden Bereichen. Er bleibt allerdings aus der engeren Völkergemeinschaft der abendländischen Christenheit ausgeschlossen und entwickelt ein eigenes, von Byzanz geprägtes Völkerrecht.4

Nach den großen Entdeckungen weitet sich der Aktionsradius der Europäer schnell auf die gesamte Erde aus. Es beginnt die europäische Expansion nach Übersee. Damit entsteht auch zwangsläufig die Frage, welche Rechtsregel für die Beziehungen zwischen den überseeischen Gemeinwesen und den europäischen Staaten gelten sollten. Einmal ist schon problematisch, ob es sich bei diesen Beziehungen um völkerrechtliche Beziehungen handelt. Zum anderen ist fraglich, ob die unterschiedlichen Formen politisch organisierter

3 Vgl. Immanuel Kant, Metaphysik der Sitten, Rechtslehre § 15, Gesammelte Werke, hrsg. von Wilhelm Weischedel, Bd. VII; Kolonialismuskritik ist auch im 3. Definitivartikel der Schrift zum Ewigen Frieden, Gesammelte Werke, Bd. XI, S. 216 f. enthalten, wo Kant ausführt: "Vergleicht man ... das inhospitale Betragen der gesitteten, vomehmlich handeltreibenden Staaten unseres Weltteils, so geht die Ungerechtigkeit, die sie in dem Besuche fremder Länder und Völker (welches ihnen mit dem Erobern derselben für einerlei gilt) beweisen, bis zum Erschrecken weit ..."

4 Vgl. Wilhelm G. Grewe, Epochen der Völkerrechtsgeschichte, Baden-Baden 1984, S. 72 ff.; Georg Stadtmüller, Geschichte des Völkerrechts, Teil 1, Bis zum Wiener Kongreß (1815), Hannover 1951, S. 54 f.; Stephan Verosta, Geschichte des Völkerrechts, in: Alfred Verdroß, Völkerrecht, 5. Aufl., S. 31 ff. (58 f.); Roberto Ago, Die pluralistischen Anfänge der internationalen Gemeinschaft, in: Völkerrecht und Rechtsphilosophie, Internationale Festschrift für Stephan Verosta zum 70. Geburtstag, Berlin 1980, S. 25 ff. (37 ff. und 47 ff.); Wolfgang Preiser, History of the Law of Nations (bis 1648), in: Encyclopedia of Public International Law, 7, 1984, S. 143 ff.; siehe auch Karl-Heinz Ziegler, Zur Einführung - Völkerrechtsgeschichte, JuS 1987, S. 350 ff. (352 f.); zur führenden Rolle von Byzanz vgl. Eichhoff, Macht und Sendung, Byzantinische Weltpolitik, 1981; Felix, Byzanz und die islamische Welt im früheren 11. Jahrhundert, 1981; zum antiken Völkerrecht vgl. etwa Bengtson, Die Staatsverträge des Altertums II: Die Verträge der griechisch-römischen Welt von 700 bis 338 v. Chr., 2. Aufl., 1975; H. H. Schmitt, Die Staatsverträge des Altertums III, die Verträge der griechisch-römischen Welt von 338 bis 200 v. Chr., 1969; Karl-Heinz Ziegler, Kriegsverträge im antiken römischen Recht, Zeitschrift der Savigni-Stiftung für Rechtsgeschichte, Bd. 102 (1985), S. 40 ff.; ders., Tradition und Wandel im Völkerrecht der römischen Spätantike, Festgabe Preiser, 1983, S. 11 ff. 
Gebilde in Ubersee, die von festgefügten Großreichen bis zu lockeren Stammesorganisationen reichen, als Völkerrechtssubjekte anerkannt werden dürfen oder ob ihnen mangels Christentum bzw. später mangels Zivilisation die Rechtsfähigkeit aberkannt werden muß.

\section{Die Lehre von der Rechtlosigkeit der Überseeischen Gebiete und die Spät- scholastiker}

Am Anfang steht dabei die Idee der christlich-abendländischen Völkerfamilie im Vordergrund. So werden in den ersten päpstlichen Bullen von 13445, 14186, 14367 und 14558, die sich auf überseeische Gebiete beziehen, Rechte der dort ansässigen Bevölkerung oder der dortigen Gemeinwesen gänzlich geleugnet. Darüber hinaus gesteht der Papst Spanien bzw. Portugal jeweils das Recht zu, die betreffenden Gebiete zu erobern. Dies kommt in der Bulle Romanus Pontifex 1455 besonders deutlich und in aller Schärfe zum Ausdruck. Die Belehungsformel faßt die verschiedenen Aspekte zusammen und verleiht den portugiesischen Königen das ausschließliche Recht, die Reiche und den Besitz der Sarazenen, Heiden und anderer Feinde Christi zu bekriegen, zu unterwerfen und sich anzueignen und ihre Bewohner in ewige Sklaverei zu führen. ${ }^{9} \mathrm{Zu}$ einer ersten umfassenden und bedeutsamen Auseinandersetzung über die Rechte der überseeischen Völker und Gemeinwesen kommt es im Zusammenhang mit dem Vorgehen der Spanier in der Neuen Welt. Nachdem bekannt wird, daß die Spanier das Reich der Azteken mit brutaler Härte zerschlagen haben, formiert sich und - und dies ist besonders bemerkenswert - in der spanischen Kirche eine breite Oppositionsbewegung.

Dabei sind die Werke der spanischen Spätscholastiker für die Entstehung des modemen Völkerrechts bahnbrechend und in ihrer Bedeutung kaum zu überschätzen. Dies wird in zahlreichen Abhandlungen über die Völkerrechtsgeschichte und die Entstehung des modernen Völkerrechts meist nicht genügend gewürdigt. Erste zentrale Figur für die nachfolgende Diskussion ist Vitoria, der die einschlägigen Fragen in beeindruckender Weise in

Bulle vom 28.11.1344, abgedruckt bei Alfonso Garcia Gallo, Las balas de Alejandro v. y el ordenaniento juridico de la expansion portuguesa y castellana en Africe y Indias, in: Anuario de historia de derecho espanol 27/28 (1957/58), S. 461 ff. (738 f.).

6 Bulle rex regnum vom 4.4.1418; abgedruckt bei Levy Maria Jardao, Bullavium patronatus Portugalliae regum in excelsiis Africae, Asiae atque Oceniae, Bd. 1, Lissavon 1868, S. 59.

7 Bulle Romanus pontifex vom 15.9.1436, abgedruckt bei Charles-Martial de Witte, Les bulles pontificales et l'expanson portugaise au Xe siècle, in: Revue d'histoire exxlésiastique 48 (1953), S. $683 \mathrm{ff} .(717 \mathrm{f}$.$) .$

8 Romanus pontifex vom 8.1.1455, abgedruckt bei Frances Gardiner Davenport, European Trearies Beaving on the History of the United States and its Dependencies, Bd. 1, Washington 1917, S. 13 ff.

9 Vgl. zu diesen päpstlichen Bullen Jörg Fist, Die europäische Expansion und das Völkerrecht, Stuttgart 1984, S. $206 \mathrm{ff}$. 
seiner Vorlesung "De indis"10 systematisiert und zu lösen versucht. Vitoria erkennt den kürzlich entdeckten Indem, also den mittelamerikanischen Indianem, Souveränitäts- und Besitzrechte zu. Sie sind nach seiner eigenen Auffassung rechtmäßige Eigentümer und Besitzer des Landes. Spanien könne weder vom Kaiser, noch vom Papst einen Titel auf das Land der Indianer herleiten. Der Kaiser sei nicht der Herr der Welt, und der Papst habe nur Befugnisse über die Christenheit und könne demnach keine Gebiete der Heiden in Amerika vergeben. Auch das Recht der Entdeckung gebe Spanien keinen legitimen Titel gegenüber den bestehenden Indianerreichen. Zwar dürfe herrenloses Gebiet von dem, der es zuerst entdeckt habe, in Besitz genommen werden, Die Gebiete in Amerika seien aber nicht herrenlos, sondern von Völkem bewohnt, denen Souveränitäts- und Besitzrechte zuständen. Auch die Weigerung der Indianer, das Christentum anzunehmen, und ihre etwaigen Verstöße gegen das Naturgesetz gäben den Christen kein Eroberungsrecht und keinen gerechten Kriegsgrund.

Auch Suàrez11, der neben Vitoria wohl bedeutsamste spanische Spätscholastiker, gesteht den Indianerreichen ausdrücklich die gleichen Rechte zu wie den christlichen Reichen. In seinem Werk wird bereits das Ideal, die Gleichheit aller Völker, besonders deutlich.12 Bei Vitoria und Suàrez erhebt demnach das Völkerrecht den Anspruch auf Universalität und bezieht daher alle Gemeinwesen in seinen Geltungsbereich ein.13 Allerdings erkennen Vitoria und Suàrez den christlichen Reichen zwei wichtige Sondertitel zu. Wenn die Verkündigung des Evangeliums behindert wird oder ein ungläubiger Fürst seinen Unterta-

10 Francisco de Vitoria, De Indis recenter inventis et de jure belli Hispanoram in Barbaros relectiones, Vorlesungen über die kürzlich entdeckten Inder und das Recht der Spanier zum Krieg gegen die Barbaren, Lateinischer Text nebst deutscher Übersetzung von Walter Schätzel, Tübingen 1952; vgl. auch Josef Soder, Die Idee der Völkerrechtsgemeinschaft. Francisco de Vitoria und die philosophischen Grundlagen des Völkerrechts, Frankfurt a.M. 1955, S. 20 ff., der Vitoria zu Recht als Begründer der modernen Lehre des Völkerrechts bezeichnet, so auch ders., Francisco Suárez und das Völkerrecht, Frankfurt a.M. 1973, S. 65 ff.; vgl. zur Bedeutung Vitorias auch Jörg Fisch (Fn 9), S. 212 ff.; Wilhelm G. Grewe (Fn 4), S. 173 ff., der m.E. die Bedeutung Vitorias für das moderne Völkerrecht unterschätzt.

11 Vgl. zur Bedeutung Suárez' für das moderne Völkerrecht vor allem Josef Soder, Francisco Suárez und das Völkerrecht, Frankfurt 1973; Jörg Fisch (Fn 9), S. 215 ff.

12 Vgl. Francisco Suárez, Tractatus tertius de charitate, Opera omnia, Bd. 23, Paris 1858, S. 747 f.; vgl. dazu auch ausführlich Josef Soder (Fn 11), S. 310 ff. m.w.N.

13 Daher gebührt Vitoria, Suárez und vielleicht noch Vásquez de Menchaca der Ehrentitel "Väter des Völkerrechts" sehr viel eher als Hugo Grotius; siehe zu der Diskussion um Hugo Grotius als "Vater des Völkerrechts" Christoph Link, Hugo Grotius als Staatsdenker, Tübingen 1983; Peter Haggenmacher, Grotius et la doctrine de la guerre juste, Paris 1983, S. 622; Wilhelm G. Grewe, Grotius Vater des Völkerrechts, in: Der Staat, 23 (1984), 161 ff.; Hartmut Schiedermair, Hugo Grotius und die Naturrechtsschule, in: Einigkeit und Recht und Freiheit, Festschrift für Karl Carstens, Köln 1985, S. 477 ff.; Karl-Heinz Ziegler, Hugo Grotius als "Vater des Völkerrechts", in: Gedächtnisschrift Wolfgang Martens, Berlin und New York 1987, S. 851 ff., der Grotius den Ehrentitel weiter zuerkennen will, aber zu Recht die Bedeutung der Spätscholastiker (S. 856) hervorhebt. 
nen die Annahme des Christentums verbietet, darf gegen die Ungläubigen Krieg geführt werden. Dies ist kein Widerspruch zu der Aussage, daß die Weigerung der ungläubigen Indianer, das Christentum anzunehmen, den Christen keinen gerechten Kriegsgrund gibt. Der gerechte Krieg in dem Sondertitel wird von Suàrez und Vitoria nicht damit gerechtfertigt, daß sich die Indianer weigern, das Christentum anzunehmen, sondern damit, daß die Mission gewaltsam behindert wird. Beide gehen also davon aus, daß das Völkerrecht die Ungläubigen verpflichte, die christliche Mission unbehindert hinzunehmen.14 Besondere Bedeutung für die nachfolgende Diskussion hat Vázquez de Menchaca15, der nicht aus kirchlichen Kreisen kommt und bereits 1564 die Frage der überseeischen Gebiete in seinen Controversiae illustres behandelt. Er lehnt die christlichen und zivilisatorischen Sondertitel ganz entschieden ab, weil er alle Menschen als frei geboren ansieht.16 Damit stellt Vázquez in gewisser Weise einen Vorläufer von Christian Wolff dar, zumal durch seine Werke die spätscholastischen Vorstellungen auch an die niederländische Universität Leiden gelangten und damit auch Hugo Grotius, der häufig, aber trotzdem irreführend als "Vater des (modernen) Völkerrechts" bezeichnet wird, beeinflußten. 17

Bereits im 18. Jahrhundert und erst recht im 19. Jahrhundert verliert das "Christentum" seine Bedeutung als die tragende Gemeinschaft des Völkerrechts. So kommt seit 1815 in den großen internationalen Verträgen die Bezugnahme auf die Christenheit als wichtiges Prinzip nicht mehr vor. 18 Es verwundert daher nicht, daß der Gedanke der Gleichheit aller Völker mit der Aufgabe bzw. Schwächung der Idee der christlich-europäischen Völkerfamilie noch konsequenter vertreten wird. Besondere Bedeutung erlangt in diesem Zusammenhang Christian Wolff 19 , der alle angeblichen christlichen, europäischen und zivilisatorischen Sonderrechte gegenüber überseeischen Gemeinwesen und Völkern ablehnt. Nach seiner Ansicht dürfen lediglich herrenlose Gebiete okkupiert werden. Herrenlos sind nach seiner Auffassung aber nur diejenigen Gebiete, die nicht von einem Volk in Besitz genommen wurden. Ein Volk setze voraus, daß sich die Menschen in einem bürgerlichen Zustand zusammengeschlossen hätten.20 Dazu reichen aber nach Auffassung von Wolff sehr lockere Organisationsformen aus. Es muß sich lediglich um einen Menschenverband handeln, der nach einer, wenn auch ungeschriebenen, Rechtordnung organisiert ist. Dabei

14 Vgl. Dieter Dörr, Die "Indian Nations and Tribes" und das Völkerrecht, JÖR n.F. 36 (1987), S. 489 ff. (491); Fisch (Fn 9), S. 217 f.

15 Vgl. zu Vásquez de Menchaca vor allem Kurt Seelmann, Die Lehre des Fernando Vásquez de Menchaca vom Dominium, 1979; siehe auch Fisch (Fn 9), S. 243 f.

16 Fernando Vásquez de Menchaca, Contoversias fundamentales y otras de más frcuente, hrsg. v. Fidel Rodriguez Alcalde, Bd. 1, Kap. 10, S. 4 f.

17 Vgl. dazu Ziegler (Fn 13), Gedächtnisschrift Martens, S. 851 ff. und oben Fn 13.

$18 \mathrm{Vgl}$. Grewe (Fn 4).

19 Siehe dazu etwa Fisch (Fn 9), S. 270 ff.

20 Vgl. z.B. Christian Wolff, Jus naturale methodo scientifica pertractum, Bd. 2, Halle 1742, Ndr. Hildesheim 1968, §§ 175-197. 
ist es gleichgültig, ob die Menschen als Nomaden leben oder seßhaft sind. ${ }^{21}$ Wolff beschreibt das Volk als Rechtszustand und greift insoweit auf eine alte philosophische Tradition zurück. Nicht der Staat, sondern das Volk als rechtlich organisiertes Gemeinwesen ist bei ihm für die Völkerrechtssubjektivität entscheidend.

\section{Vattel und die Zivilisationsidee}

Diese These von der grundsätzlichen Gleichheit aller Völker setzt sich aber in der Völkerrechtslehre des späten 18. und vor allem des 19. Jahrhunderts nicht durch. Es entsteht vielmehr die Idee der Gemeinschaft der "zivilisierten" Staaten. Auf dieser Grundlage wird die Rechtsfähigkeit der überseeischen Gemeinwesen zunehmend verneint. Die meisten Abhandlungen betreffen interessanterweise Nordamerika. Dies hat auch und vor allem historische Gründe. Die Stellung der amerikanischen Gemeinwesen war teilweise sehr stark, was in Europa noch heute zum großen Teil unbekannt ist. So stellte die Irokesenföderation lange Zeit einen bedeutenden Machtfaktor dar, den die konkurrierenden europäischen Mächte - also England und Frankreich - als Bündnispartner gewinnen wollten. Nach Meinung zahlreicher englischer und amerikanischer Historiker hat die Irokesenföderation den Kampf um die Vorherrschaft zwischen Frankreich und England entschieden bzw. nachhaltig beeinflußt.22

Die Lehre von der Rechtlosigkeit der überseeischen Gebiete und der Gemeinschaft der zivilisierten Staaten bzw. Nationen geht zum großen Teil auf den Schweizer Emer de Vattel zurück, der der wichtigste Gegenspieler von Wolff ist. Zunächst übernimmt Vattel23 in fast allen Punkten Wolffs Auffassung. Der Unterschied besteht in der Zivilisationsidee. Vattel entwickelt in diesem Zusammenhang die Lehre vom "Nutzen der Menschheit", die vor allem dazu dienen soll, das Vorgehen Großbritanniens in Nordamerika zu rechtfertigen. Danach haben die jeweiligen Völker nur das Recht, sich ein Land für den Nutzen, den sie daraus ziehen, anzueignen. Sie dürfen aber nicht verhindern, daß andere Völker Nutzen aus

21 So zu Recht Stephan Verosta, Der Vertrag zwischen Portugal und dem Marathen-Staat von 1779 europäisches oder universelles Völkerrecht, Festgabe Preiser, Baden-Baden 1983, S. 95 ff.

22 Siehe dazu z.B. Georg Frederici, Der Charakter der Entdeckung und Eroberung Amerikas durch die Europäer, Neudruck der Ausgabe 1925-1936, Bd. 2, Osnabrück 1969, S. 318 ff. m.w.N.; Felix S. Cohen, Handbook of Federal Judian Law, Washington 1962, S. 416 f.; Francis Jennings, The ambiguous Iroquois empire, New York u.a. 1984; zur ungeschriebenen Verfassung der Irokesenföderation vgl. z.B. Gaianerekowa, Das Große Friedensgesetz des Langhaus-Volkes (Irokesenbund), redigiert und hrsg. von Frank / Wagner, Saarbrücken 1988; siehe dazu auch den Brief von Benjamin Franklin an Mr. Parker vom 20. März 1751, auszugsweise abgedruckt in Henry $C$. Dennis, The American Indian 1492 - 1976, Dobbs Ferry 1977, S. 14.

23 Emer de Vattel, Le droit des gens ou principes de la loi naturelle appliqués à la conduite et aux affaires des Nations et des Souverains, hrsg. von Albert de Lapradelle, Bd. 1, Washington 1916, S. $1-16,18,81,208 \mathrm{f}$. 
dem gleichen Land ziehen. Das Land muß also nach Auffassung von Vattel nach Bedürfnissen geteilt werden. Dies gibt den eng zusammenlebenden zivilisierten Völkern das natürliche Recht, sich das Land von Nomaden anzueignen, weil jene mit dem Land verschwenderisch umgehen und es nicht effektiv nutzen. Dabei entwickelt Vattel eine Stufenleiter der Zivilisation. Völker, die das Land nicht bebauen und lieber vom Raum leben, wie die alten Germanen und manche Tataren, verdienen es nach seiner Ansicht, wie wilde und schädliche Tiere ausgerottet zu werden. Nomadenvölkern, zu denen Vattel die nordamerikanischen Indianer zählt, darf nur ein Teil ihres Landes von den seßhaften Völkem Europas weggenommen werden, um das Land im Interesse der gesamten Menschheit besser zu nutzen.24 Vattel bezeichnet die Indianer bereits als Wilde und gesteht den Europäern das Recht zu, diese Wilden zurückzudrängen und in engere Grenzen einzuschränken. Eine Zustimmung der Wilden zum Landverlust, etwa in Form von Verträgen, ist nach seiner Meinung zwar durchaus wünschenswert, aber völkerrechtlich nicht notwen$\operatorname{dig} .25$

\section{Die Lehre von den rechtlosen Wilden}

Von dieser Lehre und der darin zum Ausdruck kommenden Zivilisationsidee ist es nur noch ein kleiner Schritt zur Auffassung von der Gemeinschaft der civilized nations des späten 18. und 19. Jahrhunderts.26 Die Völkerrechtsgemeinschaft wird dabei mit der Zivilisationsgemeinschaft gleichgesetzt. Insoweit scheint noch kein großer Unterschied zur Auffassung von Wolff zu bestehen, der ja das Volk ebenfalls als Rechtszustand beschreibt und somit auch eine gewisse Zivilisationsidee zugrunde legt. Jedoch werden von den Vertretern der Lehre von der Gemeinschaft der civilized nations alle außereuropäischen Völker per se als unzivilisiert angesehen und lediglich die europäischen Staaten und später die USA zu den zivilisierten Nationen gerechnet.27

Der in diesem Zusammenhang maßgebliche Zivilisationsbegriff ist allerdings mit dem deutschen Wort Zivilisation nur recht ungenau umschrieben, da in der maßgeblichen englischen und französischen Sprache das Wort civilization bzw. civilisation sowohl die Kultur als auch die technisch-industriellen Errungenschaften meint. Allerdings ist auch der englische Begriff eng mit dem technisch-industriellen Fortschritt verbunden. Wegen des angeblichen Fehlens von technisch-industriellen Errungenschaften wird den eingeborenen Völkem in Amerika, Afria und Asien auch die Kultur und damit civilization abgesprochen. Dies hat zur Folge, daß sie im Völkerrecht keine Rechtsfähigkeit besitzen und ihnen gegen-

\footnotetext{
24 Siehe oben Fn 23.

25 Siehe oben Fn 23.

26 Vgl. zu dieser Lehre vor allem Fisch (Fn 9), S. 349 ff.; Grewe (Fn 4), S. 638 ff.

27 Siehe oben Fn 26.
} 
über eine Völkerrechtsverletzung gar nicht möglich ist. John Stuart Mill bringt diese Lehre auf einen kurzen und prägnanten Nenner mit dem berühmten Satz: "Barbariens have no right as a nation." 28

Legt man diese Lehre zugrunde, dürfen alle überseeischen Gebiete frei okkupiert werden, da sie völkerrechtlich herrenlos sind. Diese Lehre beruht selbstverständlich auf den falschen Vorstellungen über die eingeborenen Völker in Amerika, Afrika und Asien, die man gemeinhin als nomadisierende Jäger, die frei und unabhängig außerhalb irgendwelcher Gemeinwesen leben, ansieht. Dies gilt vor allem für Nordamerika, und dazu haben Rousseau29, Tocqueville 30 , Cooper 31 und später auch Thoreau 32 mit ihrem Bild vom edlen Wilden, aus dem aber schnell der Barbar und später der "Rote Teufel" wird, maßgeblich beigetragen. Dieses Bild ist gerade in Nordamerika auch heute noch für viele Vorstellungen prägend.

Die Tatsachen waren aber auch in Nordamerika anders. So wird die Organisation der Irokesenföderation von Benjamin Franklin als Vorbild für eine zu schaffende Union der englischen Kolonien, die später mit der Gründung der Vereinigten Staaten verwirklicht wird, angeführt.33 Die Irokesen nehmen heute für sich in Anspruch, mit ihrer ungeschriebenen Verfassung die Freiheitsrechte und die Gewaltenteilung lange vor den Vereinigten Staaten und den Europäem verwirklicht zu haben und eines der ältesten Kulturvölker der Erde zu sein. 34 Es ist auch unbestritten, daß nicht nur die Irokesen, sondern auch zahlreiche andere nordamerikanische Indianer und afrikanische Stämme seßhaft waren, in befestigten Orten wohnten und eine hochentwickelte Landwirtschaft besaßen. 35 Das Bild vom nomadisierenden, edlen Wilden scheint allenfalls bei oberflächlicher Betrachtung für die Prärieindianer und die Jägerstämme in Afrika zuzutreffen. Allerdings ist in Amerika die Kultur der Prärieindianer erst nach und durch die europäische Einwanderung entstanden. Durch den Druck der Expansion nach Westen wurden die Indianer zunehmend in die Plains abgedrängt, aus Jägern und Ackerbauern wurden reine Jäger wegen des Wildreichtums in den

28 John Stuart Mill, A few words on non-intervention, in: Dissertations and Discussions, Political and Historical, Bd. 3, London 1867, S. 153 ff. (168).

29 Jean-Jacques Rousseau, Contract social ou principe du droit politique, 1762.

30 Alexis de Ticqueville, De la Democratie en Amérique, Bruxelles 1840, Bd. 1, S. 45 f.; Bd. III, S. 125-130, 146.

31 James Fenimore Cooper, The last of the Mohicans, 1862.

32 Henry David Thoreau, Walden, 1854.

33 Vgl. Benjamin Franklin, Brief an Mr. Parker vom 20. März 1751, abgedr. in Dennis (Fn 22), S. 14.

34 Vgl. Ein Ruf zur Einsicht, Die Botschaft der Irokesen an die wesentliche Welt, Bühl 1984, S. 14 und $24 \mathrm{f}$.; vgl. dazu die ungeschriebene Verfassung in der allerdings historisch umstrittenen ins Deutsche übertragenen Fassung, Gaianerekowa, Das große Friedensgesetz des Landhaus-Volkes (Irokesenbund)(Fn 22).

35 Dazu Frederici (Fn 22), Bd. 2, S. 326 ff. m.w.N.; Robert F. Sayre, Thoreau and The American Indians, Princeton 1977, S. 1 ff. 
Prärien und den Pferden, die von den Spaniern nach Amerika gebracht wurden. Allerdings lebten auch diese Jägerstämme in hochentwickelten Gemeinwesen mit föderalen Organisationsstrukturen. 36 Es bleibt aber festzuhalten, daß das Bild vom edlen Wilden von der Völkerrechtslehre aufgenommen und dazu mißbraucht wurde, die außereuropäischen Völker zu entrechten.

Obwohl die Lehre von der Gemeinschaft der civilized nations, die die außereuropäischen Gemeinwesen ausschloß, auf falschen Voraussetzungen beruhte, scheint sie Grundlage des geltenden Völkerrechts gewesen zu sein, wenn man die Darstellungen der europäischen und auch der deutschen Völkerrechtler 37 zugrunde legt. Fragt man die heutigen Werke zur Völkerrechtsgeschichte, etwa auch das ansonsten vorzügliche Werk von Grewe, so waren die außereuropäischen Gemeinwesen aus der Völkerrechtsgemeinschaft ausgeschlossen. Die dort lebenden Völker wurden als "Wilde" ausgewiesen, ihnen gegenüber waren Völkerrechtsverletzungen gar nicht möglich, und ihr Gebiet durfte als herrenlos okkupiert werden. Dies mag man, wenn man diesen Autoren folgt, moralisch bedauem, es war aber geltendes Völkerrecht. 38

\section{Die Staatenpraxis unter besonderer Berücksichtigung Nordamerikas}

Allerdings kommen demjenigen, der sich mit den Darstellungen der Völkerrechtsgeschichte nicht begnügt, sondern die Quellen zurate zieht, bereits nach kurzer Zeit große Bedenken, ob die Lehre von der Gemeinschaft der civilized nations jemals geltendes Völkerrecht darstellte. Für das Völkerrecht ist auch und vor allem die Staatenpraxis von entscheidender Bedeutung. 39 Die Praxis der europäischen Staaten und der USA, mit außereuropäischen Gemeinwesen Verträge zu schließen, steht zu der Lehre vom schrankenlosen Okkupationsrecht und von der Rechtlosigkeit der sogenannten Wilden von Anfang an in einem bemerkenswerten Widerspruch. Die Existenz dieser Verträge wird noch heute in der europäischen völkerrechtlichen Literatur nur vereinzelt zur Kenntnis genommen. Leider berücksichtigt auch Grewe in seinen "Epochen der Völkerrechtsgeschichte" diese Verträge nur ganz am Rande und gelangt daher zu dem unhaltbaren Schluß, durch solche Verträge seien allenfalls Eigentumsrechte, niemals aber Souveränitätsrechte übertragen worden. 40 Die mangelnde Berücksichtigung dieser Verträge mag auch darin begründet sein, daß zahlreiche Vertrags-

36 Vgl. z.B. zu den Sioux (Lakota, Dakoto und Nakoto), die zu Unrecht als Paradebeispiel für die nomadisierenden Wilden gelten, Royal B. Hassrik, Das Buch der Sioux, 1982; zu den Cheyenne Indians siehe Karl H. Schlesier, Die Wölfe des Himmels, Welterfahrung der Cheyenne, Köln 1985.

37 Siehe dazu vor allem Grewe (Fn 4), S. 638 ff. m.w.N.

38 Siehe oben Fn 37.

39 So z.B. zu Recht Hartmut Schiedermair, Effektive Herrschaftsgewalt und Rechtsfähigkeit im Völkerrecht, JA 1984, S. 638 ff. (639); JCJ Reports 1949, 179.

40 Grewe (Fn 4), S. $638 \mathrm{ff}$. 
sammlungen die sogenannten Kolonialverträge nicht enthielten. Dies ist aber nunmehr anders, und die "Consolidated Treaty Series" haben nahezu alle Kolonialverträge berücksichtigt und aufgenommen. 41

Ein Studium dieser Verträge macht deutlich, daß allein die Vereinigten Staaten nahezu 400 Verträge mit den sogenannten Indian nations and tribes 42 geschlossen haben. Diese Praxis, mit den Indian nations and tribes durch Verträge wie mit anderen souveränen Staaten zu verkehren, änderte sich erst mit dem Gesetz vom 3. März 187143, das es ausdrücklich verbot, in Zukunft mit den Indian nations and tribes innerhalb des Territoriums der Vereinigten Staaten Verträge zu schließen. Auch Großbritannien schloß mit den indianischen Gemeinwesen in Nordamerika zahlreiche Verträge. Das gleiche Bild zeigt sich in Afrika. Belgien, Frankreich, Deutschland und Großbritannien verkehrten mit den afrikanischen Stämmen auf der Grundlage von Verträgen und versuchten vertragliche Titel auf das Land zu erwerben.44 In gleicher Weise wurden in Südostasien sowohl von Großbritannien als auch von den Niederlanden mit den dortigen Gemeinwesen zahlreiche Verträge geschlossen, die vor allem Landabtretungen zum Gegenstand hatten. 45

Besonders deutlich haben die Vereinigten Staaten von Beginn an zum Ausdruck gebracht, daß sie nicht davon ausgehen, das Gebiet der sogenannten Wilden, nämlich der Indianer, frei okkupieren zu dürfen. Bereits im Gesetz vom 3. März 178946 ist festgelegt, daß den Indianern ihr Land und ihr Eigentum nicht ohne ihre Einwilligung genommen werden darf, mit Ausnahme von gerechten und rechtmäßigen Kriegen, denen der Kongreß zugestimmt haben muß. Außerdem bestimmt Sektion 4. des ersten non intercourse act von 179047, daß kein Landverkauf, den ein Indianer oder ein indianisches Gemeinwesen innerhalb der Vereinigten Staaten getätigt hat, wirksam ist, wenn er nicht durch einen Vertrag mit der Regierung der Vereinigten Staaten erfolgte. Schon mit der Aussage, daß gegen die Indianer nur gerechte Kriege geführt werden dürfen, wird die Völkerrechtsfähigkeit der Eingeborenen insoweit bejaht und ihnen Kombattantenstatus zuerkannt.

Die gesamten Kolonialverträge, deren Form und Inhalt für eine Zuordnung zum Völkerrecht sprechen, widerlegen die Lehre vom freien Okkupationsrecht. Ihre Einordnung führte

41 Vgl. zu den Fundstellen Parry, The Consolidated Treaty Series, Special Chronologue 1648-1920, Vol 1, 2.

42 In den Verträgen wurden die indianischen Gemeinwesen anfangs meist als "Nations" und später auch als "Tribe" bzw. "Band" (kleinere Untergruppen) bezeichnet; vgl. dazu Dörr (Fn 14) mit Beispielen in Fn 26, 27.

4316 Stat. 566.

$44 \mathrm{Vgl}$. zu den Fundstellen der einzelnen Verträge Parry (Fn 41).

45 Siehe oben Fn 44.

46 Siehe dazu Dörr (Fn 14), S. 496.

47 1. Stat. 137. 
in der Völkerrechtslehre des 19. Jahrhunderts zu zahlreichen Schwierigkeiten und Ungereimtheiten und macht deutlich, daß die Lehre vom freien Okkupationsrecht und die Rechtsfigur der Wilden im wesentlichen dazu diente, Vertragsbrüche nachträglich zu rechtfertigen und sich daher erst nach Beendigung der europäischen Expansion in der zweiten Hälfte des 19. Jahrhunderts zunehmend durchsetzte. 48

Interessant ist vor allem, daß die Lehre von der Herrenlosigkeit der Gebiete, die von Wilden bewohnt werden, und dem freien Okkupationsrecht der europäischen Staaten gerade in Nordamerika von der Staatenpraxis nicht zugrunde gelegt wird, wo man die Prototypen der Wilden 49 entdeckt zu haben glaubte.

In diesem Zusammenhang ist aber zu bemerken, daß die generelle Anerkennung des Rechts der indianischen Gemeinwesen auf ihr Land und die Praxis, mit diesen Gemeinwesen durch Verträge zu verkehren, nicht zu falschen Schlüssen führen darf. Dies bedeutet nicht, daß die Ureinwohner in Nordamerika und speziell in den Vereinigten Staaten besonders rücksichtsvoll behandelt wurden; es ist das Gegenteil gerade für die zweite Hälfte des 19. Jahrhunderts 50 richtig.

Von Beginn der europäischen Besiedelung Nordamerikas an sind verschiedene Phasen der Indianerpolitik festzustellen: Zunächst wurden die indianischen Gemeinwesen von 1533 (Beginn der Besiedelung der Ostküste) an als voll souveräne Nationen behandelt.51 Die Festlegung der Grenzen zwischen den Kolonien und den indianischen Föderationen an der Ostküste und der Erwerb des Grundes erfolgte durch Vereinbarungen zwischen den Vertretern der Stämme und denen der englischen Krone, zu Beginn auch der Niederlande. Die Vereinbarungen bezeichnete man als Verträge und sah sie als international bindend an.52 Dies lag auch daran, daß die indianischen Stämme und Föderationen eine emstzunehmende militärische Bedrohung darstellten, und dies wird etwa daraus erhellt, daß König Ludwig XIV. nicht etwa England, sondern die Irokesenföderation als den gefährlichsten Gegner Frankreichs in der Neuen Welt bezeichnete. 53

48 Vgl. dazu Fisch (Fn. 9), S. 349 ff.

49 Siehe oben Fn 29-32.

50 Siehe dazu etwa Dee Brown, Bury My Heart at Wounded Knee, New York 1970, m.w.N.

51 Siehe dazu Felix Cohen, Handbook on Indian Law, 1982; W. Cole Durham, Indian Law in the Continental United States: An Overview, Law \& Anthropology, Internationales Jahrbuch für Rechtsanthropologie, Bd. 2 (1987), S. 93 ff. (94 ff.).

52 Durham (FN 51), S. 94; Cohen (Fn 51), S. 53; Bernd C. Peyer, Bürger - mehroder weniger, Indianer und das Gesetz in den USA, in: Peter R. Gerber, Vom Recht, Indianer zu sein, Zürich 1986, S. $103 \mathrm{ff}$. (104).

$53 \mathrm{Vgl}$. Frederici (Fn 22), Bd. 2, S. $364 \mathrm{f}$. 
Nach dem Sieg über die Franzosen im Siebenjährigen Krieg - in Nordamerika French and Indian War - und unter dem Eindruck des nachfolgenden Aufstandes von 18 indianischen Nationen unter Pontiac verkündete Georg III. in seiner Royal Proclamation von 176354, daß die Indianer ein uneingeschränktes Recht auf das von ihnen bewohnte Land besäßen und erklärte den Kamm der Appalachen zur Grenzlinie zwischen dem indianischen Gebiet und den englischen Kolonien. Dies sollte auch dazu dienen, die Kontrolle über die Kolonien zu behalten und eine ungeregelte Ausweitung der Siedlungen in das Landesinnere zu verhindern. Diese Royal Proclamation stieß auf den erbitterten Widerstand einiger auf Expansion gerichteter Kolonien und wird von englischen Historikem als eine wichtige Ursache für die Entfremdung zwischen den Kolonien und dem Mutterland angesehen, die letztendlich zur Unabhängigkeitserklärung führte. 55

Während und auch nach dem Unabhängigkeitskrieg übernahm die USA die Praxis des Vertragsschließens. Die indianischen Stämme stellten wegen des britisch-amerikanischen Gegensatzes weiterhin einen wichtigen Faktor dar, und beide Seiten versuchten, die jeweiligen indianischen Gemeinwesen auf ihre Seite zu ziehen. Dabei kam es auch nach dem Unabhängigkeitskrieg immer wieder, vor allem im Nordwesten infolge der anhaltenden Expansion (Ohiogrenze), zu kriegerischen Auseinandersetzungen zwischen den USA und verbündeten indianischen Stämmen56, in deren Folge die Grenzen durch Verträge neu festgelegt und allmählich nach Westen verschoben wurden. Die Indianer sympathisierten in dieser Zeit fast ausschließlich mit den Briten.57

Nach dem Krieg von 1812, in dem lediglich die mit England verbündeten nordwestlichen Indianerstämme unter Tecumseh58, dem wohl bedeutendsten indianischen Führer, die Eroberung Kanadas durch die USA zu Beginn des Krieges verhinderten, wurde durch den Vertrag von Gent (1814)59 die Vormachtstellung der USA gegenüber den Indianem begründet. Die Briten zogen sich ganz nach Kanada zurück.

Nunmehr bedeuteten die indianischen Gemeinwesen keine emsthafte militärische Bedrohung mehr für die USA. Die USA verkehrten mit diesen Gemeinwesen zwar weiter durch Verträge. Diese Verträge wurden nun aber zunehmend dazu eingesetzt, indianische Landrechte zu löschen und die "Wilden" umzusiedeln, sogenannte Removal Policy60. Eine verhängnisvolle Rolle spielte hier wiederum die Zivilisationsidee. So setzte sich vor allem

54 Vgl. dazu Douglas Sanders, Aboriginal Rights in Canada, An Overview, Law \& Anthropology, Bd. 2 (1987), S. 177 ff. (180 f.).

55 So z.B.B.Peyer (Fn 52), S. 104.

56 Vgl. zu dem Krieg um die Ohiogrenze 1789 - 1795 z.B. Glenn Tucker, Tecumseh, 1956, S. 50 ff.

57 Siehe oben Fn 56 und Die Wunden der Freiheit, 1983, S. 53 ff.

58 Vgl. dazu Tucker (Fn 56).

59 Vgl. oben Fn. 54.

60 Vgl. dazu Peyer (Fn 52), S. 105 ff. 
unter der Regierung des sogenannten Grenzerpräsidenten Jackson ${ }^{61}$ die Auffassung durch, daß ein friedliches Zusammenleben zwischen den "wilden Indianern" und den "zivilisierten Weißen" nicht möglich sei. Daher müßten alle Indianer in dem Gebiet westlich des Mississippi getrennt von den Weißen leben. Zu diesem Zweck sollten Verträge mit ihnen ausgehandelt und ihnen für ihr gegenwärtiges Gebiet Länder im heutigen Kansas und Oklahoma übertragen werden. 62

Den indianischen Gemeinwesen, paradoxerweise gerade und vor allem den von den USA selbst als Five civilized Tribes bezeichneten, hochentwickelten und der europäischen Lebensweise angepaßten südlichen Stämmen wurden mit militärischem Druck solche Verträge aufgezwungen, wobei vor allem die Vertreibung der Cherokee aus Georgia einen traurigen Höhepunkt darstellte. Im Verlauf dieser Vertreibung kamen ca. zwei Drittel der Cherokees ums Leben.63 Während der Removal Phase versuchte der US-Supreme Court die Rechtsstellung der Indianer auf deren Klagen hin zu klären.

\section{Die Rechtsprechung des US-Supreme Court}

So hat der US-Supreme Court in den Jahren 1823 - 1832 in drei wichtigen Entscheidungen64 zum Status der indianischen Gemeinwesen und deren Verhältnis zu den USA Stellung genommen. Diese Entscheidungen sind jeweils von Chief Justice Marshall abgefaßt. Er gestand den Indianern ein eigenes Recht auf ihr Land zu65 und bezeichnete die Indian nations and tribes als domestic dependant nations66, also inländische, abhängige Nationen. Die einzelnen Begriffe werden in den Entscheidungen "Cherokee nation versus Georgia" 183167 und "Worcester versus Georgia" 183268 näher erläutert.

So weist Marshall zunächst darauf hin, daß die indianischen nations and tribes Staaten seien und besondere von anderen getrennte politische Einheiten bildeten. Alle europäischen Staaten und die Vereinigten Staaten hätten die indianischen Stämme und Nationen seit der Besiedlung Nordamerikas als Staaten behandelt. Zahlreiche Verträge würden sie als Völker anerkennen, die in der Lage seien, kriegerische und friedliche Beziehungen zu unterhalten.

61 Andrew Jackson war Präsident von 1829 - 1837.

62 Siehe Peyer (Fn 52), S. 106 ff.

63 Vgl. Die Wunden der Freiheit (Fn 57), S. 104 ff.

64 Graham's Lessee v. M'Intosh, 8 Wheaton 543 (1823); Cherokee Nation v. Georgia, 5 Peters 1 (1831); Worcester v. Georgia, 5 Peters 512 (1932).

658 Wheaton 543, dazu Dörr (Fn 14), S. 496 f.

665 Peters 1, dazu Dörr (Fn 14), S. 498.

675 Peters 1.

685 Peters 512. 
Allerdings sei das Territorium einer Indian nation bzw. eines Indian tribe kein Ausland gegenüber den Vereinigten Staaten. Dies folge aus dem Entdeckungsrecht, das von allen europäischen Staaten anerkannt sei und in den Verträgen zwischen diesen Staaten gebilligt werde. Dieses Entdeckungsrecht gebe den USA als Entdeckerstaat im Verhältnis zu allen anderen europäischen Staaten einen exklusiven Titel, das entdeckte Land von den Indianem zu erwerben. Úber ihren Boden dürften die Indianer also nicht nach Belieben verfügen, sondern diesen ausschließlich den USA übertragen.

Schließlich seien die indianischen Gemeinwesen auch abhängig von den Vereinigten Staaten. Dies würden sie in den meisten Verträgen selbst anerkennen, da sie sich unter den Schutz der Vereinigten Staaten begeben hätten. Zudem stünden die indianischen Gemeinwesen mittlerweile in einem Stadium der Unmündigkeit. Ihre Beziehungen zu den Vereinigten Staaten glichen denen eines Mündels zu seinem Vormund.69 In diesem Zusammenhang erkennt man unschwer die Zivilisationsidee, die ein großer Teil der Völkerrechtslehre, insbesondere Vattel, im 18. und 19. Jahrhundert vertritt.

Allerdings hat Richter Marshall die Bedeutung der Aussage, da $\beta$ es sich bei den indianischen Gemeinwesen um abhängige Nationen handle, in der zweiten Entscheidung wesentlich eingeschränkt. Hier stellt er ausdrücklich fest, daß sich an der Unabhängigkeit und dem Recht der indianischen Gemeinwesen auf Selbstregierung bzw. Selbstbestimmung nichts dadurch ändere, daß sich die Indianer in den Verträgen zunächst unter den Schutz Großbritanniens und dann unter den Schutz der USA begeben hätten. Nach einem anerkannten Grundsatz des Völkerrechts verliere eine schwächere Macht ihre Unabhängigkeit und ihr Recht auf Selbstregierung nicht dadurch, daß sie sich mit einer stärkeren Macht verbünde und ihren Schutz in Anspruch nehme.70 Damit wird vor allem in der zweiten Grundsatzentscheidung die Völkerrechtsfähigkeit der indianischen Gemeinwesen, der sogenannten Wilden, ausdrücklich bejaht. Allerdings dürfen diese indianischen Gemeinwesen Außenbeziehungen nur zu dem europäischen Entdeckerstaat bzw. nunmehr zu den Vereinigten Staaten und zu keinem anderen Staat unterhalten. Diese Einschränkung folgt nach Ansicht des USSupreme Court aus dem Entdeckungsrecht, das zwar mit den Vorstellungen des Naturrechts kaum zu vereinbaren sei, aber geltendes Völkerrecht darstelle und das die Indianer hinnehmen müßten.71

Einerseits wird damit den indianischen Gemeinwesen und demnach einem Teil der sogenannten Wilden die Völkerrechtssubjektivität ausdrücklich zuerkannt. Diese unterliegt andererseits aber besonderen Beschränkungen. Es bleibt demnach dabei, daß die Beziehungen zwischen den Vereinigten Staaten und den indianischen Gemeinwesen nach Ansicht

69 Siehe Dörr (Fn 14), S. 498.

70 Siehe Dörr (Fn 14), S. 499.

71 Dazu Dörr (Fn 14), S. 499 f. 
des US-Supreme Court sowohl innerstaatliche als auch völkerrechtliche Elemente aufweisen. Die Stellung der Vereinigten Staaten als Treuhänder der Indianer mit Gesetzgebungsbefugnissen, also ihre Vormundschaftsstellung, wurde von dem US-Supreme Court niemals in Frage gestellt. Demnach deutete und deutet der US-Supreme Court auch heute noch die Beziehung zwischen den USA und den Indiantribes and nations als eine Gemengelage von Völkerrecht und innerstaatlichem Recht.

Es bleibt aber festzuhalten, daß die Staatenpraxis in Nordamerika bis zur ersten Hälfte des 19. Jahrhunderts die Völkerrechtsfähigkeit der sogenannten Wilden belegt. Diese wurden von Anfang an als fähig angesehen, kriegerische und friedliche Beziehungen zu unterhalten. Außerdem haben die europäischen Mächte und später die USA von Beginn an mit den Indianem Verträge völkerrechtlichen Inhalts auf der Basis der Gleichordnung geschlossen. Mit dieser Praxis haben die Europäer zum Ausdruck gebracht, daß sie die sogenannten Wilden als willens und in der Lage ansehen, ihre vertraglichen Verpflichtungen zu erfüllen. Diese Einschätzung war auch durchaus zutreffend, da in Nordamerika die sogenannten Wilden den Grundsatz pacta sunt servanda stets peinlich genau eingehalten und sich die angeblich zivilisierten Europäer und US-Amerikaner nicht gerade durch Vertragstreue ausgezeichnet haben. 72

\section{Die weitere Expansion der USA und die Stellung der Indianer}

Die Grenzlinie zwischen Indianern und Weißen wurde in der Folgezeit immer weiter nach Westen verschoben und den verschiedenen Stämmen weitere Verträge aufgenötigt, in denen diese häufig so lange Land abtraten, bis nichts mehr abzutreten war. Lediglich der Sioux Nation und den mit ihnen verbündeten Northern Cheyenne und Arapahoes gelang es nach einem erbitterten Krieg (Red Coud War)73, noch 1868 den USA einen Vertrag, den Fort Laramie Treaty 74 , abzutrotzen, der ihnen ihr Gebiet weiter garantierte und mit denen die Sioux ihre Position durchsetzten.

Da sich bestehende Verträge auch nach dem Zusammenbruch des bewaffneten Widerstandes der Prärieindianer (1890) nicht ohne weiteres ändern ließen, ging der Kongreß dazu über, sich mit seinen Gesetzen über die Verträge hinwegzusetzen. So wurde etwa der Sioux

72 Es gibt wohl kaum einen Vertrag mit einer Indian Nation, den die USA längere Zeit eingehalten haben.

73 Vgl. dazu Dee Brown (Fn 50), Kap. 6; eine interessante Darstellung aus indianischer Sicht, Black Elk, Ich rufe mein Volk, Aufzeichnung von J. Heilhard, übersetzt von S. Lang, 1982, S. 18 ff.; siehe auch D. Robinson, A History of the Dakota or Sioux Indians, 1904, S. $356 \mathrm{ff}$. 136 CTS 92. 
Nation bereits 1877 ihr Gebiet (Black Hills) zum großen Teil gesetzlich aberkannt ${ }^{75}$, weil sie sich gegen die Invasion in ihr vertraglich für immer gewährleistetes Gebiet unter Führung von Sitting Bull und dem genialen militärischen Taktiker Crazy Horse zur Wehr gesetzt hatten (Custer Schlacht, Krieg um die Black Hills)76.

Uber diese Praxis, die Verträge abzuändern oder aufzuheben, entschied der US-Supreme Court im Jahr 1903 auf Klage des Indianerhäuptlings Lone Wolf77. Einleitend stellte der US-Supreme Court fest, daß entsprechend der Treuhandthese der Kongreß aus seiner Treuhandstellung die Macht habe, zum Besten der Indianer für diese Gesetze zu erlassen. Die Gerichte seien wegen der beruhmten political question doctrine nicht befugt, die Vereinbarkeit solcher Gesetze mit Verträgen zu überprüfen. Später hat der US-Supreme Court die Treuhandstellung erheblich eingeschränkt und prüft heute, ob der Kongreß eine Verwaltung zum Besten der Indianer überhaupt vomimmt. Die ursprüngliche Rechtsprechung wurde in der Folgezeit dazu benutzt, nahezu sämtliche vertraglichen Rechte der Indianer durch Gesetz "zum Besten der Wilden" zu vermindern oder aufzuheben und Autonomie der Stämme vollständig zu beseitigen. Maßgeblich war dabei auch der General Allotment Act von 1887. Die Indianer sollten durch Beseitigung der Autonomie vollständig in die USA integriert werden. Daher wurde ihnen 192478 auch die amerikanische Staatsangehörigkeit zuerkannt. Dies war vorher nur für bestimmte Indianer der Fall.

1934 wurde unter Roosevelt die Indianerpolitik wegen vollständigen Scheitems dieses Integrationsversuchs wiederum geändert. Es kam auf Initiative von Roosevelt zur Verabschiedung des Indian Reorganization Act79, der die indianische Selbstverwaltung wiederherstellte und das zerstückelte Land der Stämıne (Reservate) wieder zusammenfaßte. Die Stammesverfassungen ergingen allerdings nach Muster der US-Bundesverfassung und entsprachen nicht immer indianischen Traditionen. Daher wurden die Stammesregierungen von vielen Indianem als. Marionetten der US-Regierung angesehen und mit Mißachtung behandelt. 80

75 Vgl. dazu 19 Stat. 176 und 19 Stat. 254; siehe zu den Vorgängen auch United States v. Sioux Nation of Indians, 100 S. 1t. 2716 (1980) at. 2723 f. und Reinhard Trink, The Black Hills are not for sale - Discrimination of Indigenous Peoples in the US Law System, Law \& Anthropology 3 (1988), S. 157 ff. (161 f.).

76 Siehe dazu BlackElk (Fn 73), S. 93 ff.; Robinson (Fn 73), S. 422 ff.; Dee Brown (Fn 50), Kap. 12.

7723 S. Ct. 216 (1903).

7843 Stat. 253.

7948 Stat. 984.

80 So Peyer (Fn 52), S. $110 \mathrm{f}$. 
Ab 1953 kam es erneut zu einem Rückschlag für das Selbstbestimmungsrecht mit der Termination Policy.81 $\mathrm{Ihr}$ Ziel war es, die indianische Selbstverwaltung stufenweise zu beenden und die Indianer angeblich dadurch zu vollwertigen US-Bürgern zu machen. Die Folgen dieser Politik waren nachgerade verheerend.

Daher entschloß man sich zu Politik der Selbstbestimmung - Self determination policy -, die in Ansätzen unter John F. Kennedy begann und die Grundlage der heutigen Behandlung der Indianer in den USA ist. Sie besagt, daß die Indianer selbst bestimmen und entscheiden dürfen, wie sie leben wollen, in von ihnen selbstverwalteten Gebieten (sogenannte Reservate) oder als "normale" US-Bürger. Reservate, die als Folge der Termination Policy aufgelöst worden waren, sind teilweise wieder errichtet, die Selbstverwaltung der Indianer durch Gesetze und Rechtsprechung erheblich gestärkt worden. Dies hat einmal seinen Grund im indianischen Widerstand (Red Power Bewegung) 82 . Zum anderen wird nunmehr die Zivilisationsidee zur Begründung der Rechte der eingeborenen Völker eingesetzt. Die indianische Kultur sei eigenständig, die Indianer hätten ein Recht darauf, ihre eigene Lebensweise, ihre Zivilisation, zu erhalten. Die sogenannte indianische Seele Amerikas wird in den USA nunmehr wiederentdeckt und stößt auf zunehmendes Interesse und Verständnis.

Wegen der mit den Indianern geschlossenen Verträge und der bestehenden den Indianern vorbehaltenen Gebiete (Reservate) muß sich der US-Supreme Court auch in der Gegenwart häufig mit der Rechtsstellung der indianischen Gemeinwesen in den USA beschäftigen. 83 Er geht weiterhin davon aus, daß die Indianerstämme auf ihrem Gebiet wie staatliche Einheiten nicht nur Personalhoheit über ihre Mitglieder, sondern auch Territorialhoheit über das ihnen zugewiesene Territorium besitzen. Diese Stämme sind nach Ansicht des USSupreme Court auch in der Gegenwart noch ein gutes Stück mehr als private, freiwillige Organisationen. Ihre Hoheitsrechte sind danach nicht lediglich abgeleitete Hoheitsrechte. Zwar besitzen die Indianerstämme auch solche abgeleiteten Hoheitsrechte, die ihnen durch die amerikanische Gesetzgebung und durch Verträge eingeräumt wurden. Daneben verfügen die Indianerstämme nach Auffassung des US-Supreme Court aber auch über eine inhärente Souveränität.84 Diese besteht noch insoweit, als ihnen hoheitliche Befugnisse nicht ausdrücklich durch Bundesgesetz oder Vertrag entzogen wurden. Sie sind also der

81 Siehe dazu Peyer (Fn 52), S. 111 f.; Wilkinson / Biggs, The Evolution of the Termination Policy (1977), 5 AM Jud LR 139 ff.

82 Vgl. dazu Die Wunden der Freiheit (Fn 57), S. 168 ff.

83 Vgl. dazu die umfassende Darstellung von Charles Wilkinson, American Indians, Time and the Law, 1987, in der die neue Rechtsprechung von 1959 - 1986 aufgearbeitet wird; dabei werden allein 80 (!) Entscheidungen des US-Supreme Court zum Indian Law aufgeführt.

84 Siehe z.B. Merrion and Bayless v. Jicarilla Apache Tribe, 102 SCT 894 (1982) at 903 ff., 908; United States v. Wheeler, 435 US 313 (1978) at 322 f.; umfassend dazu Wilkinson (Fn 83), S. $54 \mathrm{ff}$. 
Rest jener nach Auffassung des US-Supreme Court früher unbeschränkten Souveränität, die die indianischen Gemeinwesen auf ihrem geweiligen Gebiet einmal innehatten.

Damit erkennt der US-Supreme Court in seiner heutigen ständigen Rechtsprechung die ehemalige Völkerrechtssubjektivität der indianischen Gemeinwesen, also der Wilden, an und geht darüber hinaus davon aus, daß Reste jener originären Hoheitsgewalt weiter fortbestehen. Demnach enthalten die Beziehungen zwischen den indianischen Gemeinwesen und den Vereinigten Staaten nach der Rechtsprechung des US-Supreme Court noch heute völkerrechtliche Elemente. 85 In der Praxis der Vereinigten Staaten kann also keine Rede davon sein, daß die eingeborenen Völker als rechtlos angesehen und ihre Gebiete als herrenlos behandelt wurden oder werden. Vielmehr gingen die USA von Anfang an davon aus, daß die sogenannten Wilden und ihre Gemeinwesen eigene Souveränitätsrechte über ihr Land besaßen und die USA dieses Land durch Verträge erwerben müßten. Eine ganz andere Frage ist in diesem Zusammenhang, daß diese Verträge mit dem immer größeren Ubergewicht der USA vor allem im 19. Jahrhundert in überaus zweifelhafter Weise zustande kamen und die USA sich ständig über die mit den indianischen Gemeinwesen geschlossenen Verträge hinwegsetzen. Aber auch diese Vertragsbrüche versuchte man dann immer wieder durch den Abschluß neuer, wenn auch mit militärischer Gewalt erzwungener Verträge zu rechtfertigen.

\section{Die Entscheidungen des IGH}

Diese rechtliche Bewertung ist keine US-amerikanische Besonderheit. Auch in Kanada wird das Verhältnis zwischen den indianischen Gemeinwesen und Großbritannien bzw. Kanada ähnlich gedeutet. Entgegen früheren Entscheidungen hat sich der Supreme Court of Canada die Rechtsprechung des US-Supreme Court teilweise zueigen gemacht und geht davon aus, daß die Indianer ein eigenes Recht auf ihr Land besaßen.86 Unklar bleibt aber bei diesen Entscheidungen weiterhin, ob dies lediglich persönliche Rechte des einzelnen Indianers waren oder den indianischen Gemeinwesen so etwas wie Souveränitätsrechte zustanden. 87

In Mittelamerika kam es ebenfalls zu vertraglichen Beziehungen zwischen Großbritannien und indianischen Gemeinwesen. So wurde bereits 1720 ein förmlicher Vertrag zwischen Großbritannien und den Mosquito- bzw. Mesquito-Indianem 88 auf der Basis voller Gleich-

85 Dazu Dörr (Fn 14), S. 504 m.w.N. in Fn 69.

86 Vgl. dazu nunmehr Stephan Marquardt, The Aboriginal Peoples of Canada and their Rights under Canadian Constitutional Law, VRÜ 1989, S. 251 ff.; siehe dazu Guerin v. The Queen, 13 Q.L.R. (4th), 321 (1984), at $334 \mathrm{ff}$.

87 Vgl. Marquardt (Fn 86), S. $262 \mathrm{ff}$.

8831 CTS 235. 
berechtigung abgeschlossen. Im Zuge der Auseinandersetzungen zwischen Nicaragua und Großbritannien kam es zum Vertrag von Managua zwischen Großbritannien und Nicaragua im Jahr 1860.89 In diesem Vertrag anerkannte Großbritannien Nicaraguas Souveränität auch über das gesamte Gebiet der Mosquito-Indianer ohne deren Einverständnis. Dafür verpflichtete sich Nicaragua, den Indianem weitreichende Autonomie zu gewähren. Nicaragua hielt diese Verpflichtungen nicht ein, und Großbritannien monierte eine Verletzung des Vertrages von Managua. Der Streit wurde 1879 dem österreichischen Kaiser Franz Joseph zur Entscheidung übergeben. Der Schiedsspruch vom 2. Juli 188190 erging eindeutig zugunsten Großbritanniens und der indianischen Autonomie. In dem Schiedsspruch wird ausgeführt, daß die Mosquito-Indianer völkerrechtliche Beziehungen zu Großbritannien unterhielten. Erst 1860 sei das bisherige völkerrechtliche Schutzverhältnis durch ein staatsrechtliches Unterwerfungsverhältnis ersetzt worden. Nicaragua hatte sich zur Rechtfertigung seines Vorgehens gegen die Indianer auch auf den Zivilisationstitel berufen. Dieser wird im Schiedsspruch zwar nicht grundsätzlich zurückgewiesen, aber als Begründung für eine schlechte Behandlung der Indianer nicht akzeptiert und als Ausrede entlarvt. In jüngster Vergangenheit hat die neue, inzwischen abgelöste sandinistische Regierung ebenfalls die Autonomie der Mosquito-Indianer seit 1982 mit brutaler Gewalt beseitigt, was zum indianischen bewaffneten Widerstand Mesura (ein Zusammenschluß der Mesquito-, Suma- und Rama-Indianer) gegen die neuen Machthaber führte. 91 Gerechtfertigt wurde das brutale Vorgehen gegen die Indianer wiedenum mit der Zivilisationsidee; allerdings diesmal unter sozialistischem Vorzeichen.

Schließlich hat auch der Internationale Gerichtshof in seiner Entscheidung über den Streitfall zwischen Portugal und der Republik Indien betreffend "Durchgangsrechte über indisches Gebiet"92 deutlich gemacht, daß er ohne weiteres von der völkerrechtlichen Wirksamkeit der Kolonialverträge ausgeht. Portugal nahm in diesem Fall nämlich Rechte gegen Indien aus einem Vertrag mit dem Marathenstaat aus dem Jahr 1779 für sich in Anspruch. Der Internationale Gerichtshof ist ohne nähere Begründung selbstverständlich von der Rechtsfähigkeit der beiden damaligen Vertragspartner ausgegangen und hat daher den Vertrag von 1779 als wirksamen völkerrechtlichen Vertrag angesehen. Dies belegt, da $\beta$ in der Staatenpraxis auch die indischen und südostasiatischen Gemeinwesen in die Völkerrechtsgemeinschaft aufgenommen und von den europäischen Mächten ausdrücklich als fähig und bereit angesehen wurden, vertragliche Rechte und Pflichten einzuhalten. 93

89121 CTS 318.

90 Abgedruckt bei La Fontaine, Pacrésie intemationale Histoire documentaire des arbitrages internationeaux, Bem 1902, S. 385 ff.; vgl. zu dem Schiedsspruch auch Fisch (Fn 9), S. 394 ff.

91 Dazu Martin Kriele, Nicaragua - das blutende Herz Amerikas, 1985.

92 ICJ Reports 1960, 5 (S. 37 f.).

$93 \mathrm{Vgl}$. Fisch (Fn 9), S. $455 \mathrm{ff}$ und $37 \mathrm{ff}$. 
Schließlich wird auch im Sahara-Gutachten ${ }^{94}$ des Intemationalen Gerichtshofs aus dem Jahr 1975 deutlich, daß selbst die Westsahara zur Zeit der spanischen Kolonisation von den Spaniem nicht als herrenlos angesehen wurde. Vielmehr schloß Spanien Verträge mit den dortigen Stämmen ab, um einen völkerrechtlichen Titel auf das Gebiet der Westsahara zu erwerben. Daraus ergibt sich, daß auch die Spanier die dort ansässigen Stämme als Völkerrechtssubjekte ansahen. Demnach wird auch in dem Sahara-Gutachten mit Recht die These zurückgewiesen, daß das Gebiet der Sahara herrenlos gewesen sei.

\section{Zusammenfassung}

Unter Berücksichtigung dieser Staatenpraxis kann man nicht emsthaft behaupten, daß im Völkerrecht des 18. und 19. Jahrhunderts sämtliche überseeischen Gebiete nach der Lehre von der Zivilisationsgemeinschaft wegen der Wildheit ihrer Bewohner als herrenlos angesehen wurden. Vielmehr haben die Europäer mit ihrer Vertragspraxis zum Ausdruck gebracht, daß sie die indianischen, südostasiatischen und afrikanischen Gemeinwesen als willens und in der Lage ansahen, ihre vertraglichen Verpflichtungen zu erfüllen. Zwar ist es richtig, daß zur Völkerrechtsgemeinschaft zunächst als geborene Mitglieder nur die christlichen und später civilized nations, also die europäischen Staaten und seit ihrer Gründung die USA gehörten. Die europäischen Mächte und die Vereinigten Staaten haben aber bereits während der europäischen Expansion zahlreiche außereuropäische Gemeinwesen als Mächte anerkannt, die in der Lage sind, völkerrechtliche Beziehungen zu unterhalten. Damit wurden die sogenannten Wilden in die Völkerrechtsgemeinschaft aufgenommen. Sie erwarben die Völkerrechtssubjektivität kraft Anerkennung.95 Die Lehre von den herrenlosen Gebieten, der Gemeinschaft der civilized nations und der fehlenden Völkerrechtssubjektivität der außereuropäischen Gemeinwesen der Wilden diente vor allem dazu, die bereits eingetretenen Vertragsbrüche nachträglich zu rechtfertigen und das Vorgehen der Europäer mit der Zivilisationsidee auch moralisch zu legitimieren. Diese Lehre stand aber von Anfang an im erkennbaren Widerspruch zur Staatenpraxis und war daher nie Grundlage des geltenden Völkerrechts. Vielmehr war für die Völkerrechtssubjektivität entscheidend, ob und inwieweit die außereuropäischen Gemeinwesen als willens und in der Lage angesehen wurden, vertragliche Beziehungen zu unterhalten und einzuhalten.

Die Frage, welches $\mathrm{Ma} \beta$ an Zivilisation und an Anerkennung des Rechts erforderlich ist, um Rechtsfähigkeit im Völkerrecht zu bejahen, ist kein lediglich historisches Problem. Sie beschäftigt das Völkerrecht ständig, und so verwundert es nicht, daß die philosophische Frage, was den Staat von einer Räuberbande unterscheide, immer wieder gestellt wurde und gestellt wird. Heute bestimmt Art. 4 Abs. 1 der UN-Satzung, daß nur solche Staaten

94 ICJ Reports 1975, 12 (S. 38 ff. und 43 f.).

95 So Cohen (Fn 51), S. 39 f. 
Mitglieder der Vereinten Nationen werden dürfen, die willens und in der Lage sind, ihre Verpflichtungen aus der Satzung zu erfüllen. Die Bedeutung dieser Bestimmung reicht weit über die Frage der Mitgliedschaft in den Vereinten Nationen hinaus. Nach richtiger Auffassung enthält Art. 4 Abs. 1 der UN-Satzung eine Definition der Rechtsfähigkeit und damit des Staates im völkerrechtlichen Sinne. 96 Staaten sind daher nur solche Gemeinwesen, die bereit und fähig sind, ihre gesamten völkerrechtlichen Verpflichtungen zu erfüllen. Der Staat wird hier, wie bei Christian Wolff, als Rechtszustand beschrieben, mit der durchaus problematischen Folge, daß ein Mindestmaß an Rechtlichkeit für die Staatsqualität unentbehrlich ist.

Welches Mindestmaß an Rechtlichkeit hier nicht unterschritten werden darf, ist schwer zu beschreiben. Die Staatenpraxis ist dabei zurückhaltend. So wurde auch Nazi-Deutschland, entgegen vereinzelten Stimmen in der Literatur97, die Völkerrechtssubjektivität nicht wegen Unterschreitung des Mindestmaßes an Rechtlichkeit aberkannt. Auch Kambodscha wurde während der Terrorherrschaft der Roten Khmer nicht aus der Völkerrechtsgemeinschaft verabschiedet. Diese Zurückhaltung ist durchaus angebracht, um den universellen Geltungsanspruch des Völkerrechts nicht zu gefährden und auf diese Weise auch das Gewaltverbot zu beeinträchtigen. Welch problematische Folgen die Verabschiedung großer Teile der Gemeinwesen aus dem Völkerrecht hat, macht die Lehre von den Wilden und der Gemeinschaft der civilized nations deutlich. Mit dieser Lehre wurde versucht, die außereuropäischen Völker zu entrechten und die Brüche der Kolonialverträge zu legitimieren. Zum Glück ist die Lehre von der Rechtlosigkeit der überseeischen Gebiete und Völker nie Bestandteil des geltenden Völkerrechts geworden, auch wenn dies in den Darstellungen der Völkerrechtsgeschichte weiterhin nicht zur Kenntnis genommen werden sollte.

96 Schiedermair (Fn 39), S. 640.

97 Vgl. etwa Hans Kelsen, American Joumal of International Law, Vol. 34 (1944), S. 689 ff. 
completely eroded, such that traditional rules were still applicable despite the absence of the social values required before.

The inclusion of the Bill of Rights in the country's Constitution in 1985 made it possible for women to pursue their rights to equality within the confines of a solid legal framework. It is within the context of this development that this paper seeks to examine the issue of women's rights to inherit land. The importance of this issue is particularly underscored by the fact that land remains one of the major means of livelihood for the majority of the Tanzanian population, half of which is comprised of women.

\section{The "Barbarians" and International Law}

\section{By Dieter Dörr}

In the course of the European expansion in the age of colonialism, the terms "barbarians" (John Stuart Mill) or "die Wilden" (Immanuel Kant) have been introduced to the rapidly developing field of modem international law in order to support the argument that the territory of the overseas dependencies of the European countries had been res nullius before colonization. The legal term "die Wilden", for example, dates back to the 18th and 19th century and stands for the "lawless freedom" of the North American Red Indians as seen by the European international lawyers.

The article explains the background of the different uses of the terms and their legal implications, starting with the papal bull of 1344, in contrast to the concept of the "civilized nations". The example of the Cherokee Federation in North America proves that the facts did not support the dogma. The Cherokee, as many other North American and African tribes, had a highly developed scheme of communal organization within their federation, which was seen, by Benjamin Franklin, as a model for the projected confederation of the English colonies.

The North American state practice took respect of these facts, as the contracts with the Indian nations and tribès were subject to the principles of international law. This state practice had great influence not only on the adjudication of the US Supreme Court, but also of the Supreme Court of Canada and of the International Court of Justice. The author comes to the conclusion that there is no factual or legal basis for the assertion that the territory of the colonies had to be considered as res nullius. 